\title{
PSICOPROFILAXIS OBSTÉTRICA Y SU INCIDENCIA EN LA DEPRESIÓN POSTPARTO
} EN MADRES ADOLESCENTES.

\section{OBSTETRIC PSYCHOPROPHYLAXIS AND ITS INCIDENCE ON POSTPARTUM DEPRESSION IN ADOLESCENT MOTHERS.}

\section{Autores: ${ }^{1}$ Georgina Azucena Romero Torres, ${ }^{2}$ Josselin Cherrez Romero y ${ }^{3}$ Genesis Thayly Barros Monserrate}

ORCID ID: https://orcid.org/0000-0002-2999-9335

${ }^{1}$ E-mail de contacto: azuromero@live.com

Articulo recibido: 3 de Abril del 2020

Articulo revisado: 20 de Abril del 2020

Articulo aprobado: 1 de Mayo del 2020

${ }^{1}$ Psicologa educativa y orientadora vocacional egresada de la Universidad Técnica de Babahoyo (Ecuador) con 15 años de experiencia dentro de la psicología. Posee una maestría en la Universidad Técnica de Babahoyo (Ecuador), mención Docencia y Currículo.

${ }^{2}$ Obstetriz egresada de la Universidad Técnica de Babahoyo (Ecuador) con 4 años de experiencia dentro de la Obstetricia. ${ }^{3}$ Obstetriz egresada de la Universidad Técnica de Babahoyo (Ecuador) con 4 años de experiencia dentro de la Obstetricia.

\section{Resumen}

El objetivo que guio el desarrollo de la investigación fue, determinar la incidencia del tratamiento de Psicoprofilaxis obstétrica en la disminución de la depresión postparto en madres adolescentes del sector La Aventura Cantón Babahoyo Provincia de Los Ríos durante el primer semestre del año 2015., para darle cumplimiento al mismo se utilizaron los métodos análisis-síntesis, inductivo-deductivo, y el hipotético-deductivo, los que permitieron, dar respuesta al objetivo que guio la investigación partir de la aplicación de los instrumentos científicos de la encuesta y la guía de observación, que posibilitaron obtener los datos necesarios acerca de las variables que fueron controladas, conteniéndose como re4sultados que la Psicoprofilaxis Obstetra influyo positivamente en la disminución de las depresión post parto en las madres adolescentes que fueron objeto de estudio.

\section{Palabras claves: Psicoprofilaxis, Obstetricia, depresión, postparto.}

\begin{abstract}
The objective that guided the development of the research was to determine the incidence of obstetric psychoprophylaxis treatment in the reduction of postpartum depression in adolescent mothers in the La Aventura sector, Cantón Babahoyo Province of Los Ríos, during the first semester of 2015 , to give it In compliance with it, the analysis-synthesis,
\end{abstract}

inductive-deductive, and hypotheticaldeductive methods were used, which allowed us to respond to the objective that guided the research from the application of the scientific instruments of the survey and the observation guide, that made it possible to obtain the necessary data about the variables that were controlled, containing as results that Obstetrician Psychoprophylaxis had a positive influence on the reduction of postpartum depression in adolescent mothers who were the object of study.

Key words: Psychoprophylaxis, Obstetrics, depression, postpartum.

\section{Sumário}

O objetivo que norteou o desenvolvimento da pesquisa foi determinar a incidência do tratamento com psicoprofilaxia obstétrica na redução da depressão pós-parto em mães adolescentes do setor La Aventura, província de Cantón Babahoyo de Los Ríos, durante o primeiro semestre de 2015, para dar-lhe Para o seu cumprimento, foram utilizados os métodos de análise-síntese, indutivo-dedutivo e hipotético-dedutivo, que nos permitiram responder ao objetivo que norteou a investigação a partir da aplicação dos instrumentos científicos do inquérito e do guia de observação, isso possibilitou a obtenção dos dados necessários sobre as variáveis controladas, contendo como resultados que a psicoprofilaxia obstétrica influenciou 
positivamente na redução da depressão pósparto nas mães adolescentes objeto do estudo.

\section{Palavras-chave: Psicoprofilaxia, Obstetrícia, depressão, pós-parto.}

\section{Introducción}

Horowitz, J. (2020) afirma que la depresión post parto, se puede producir hasta un año después de haber dado a luz, según datos estadísticos de la OMS, el mayor porcentaje de privacidad en aparecer la depresión postparto es aproximadamente a los 3 meses del nacimiento del niño, lo que provoca trastornos y malestar en las madres adolescentes y el núcleo familiar, que afecta la salud física y mental del recién nacido, David, D. (2020) define a la depresión como el estado de tristeza, melancolía, infelicidad, que incide negativamente en la autoestima de la mujer en la etapa de gestación. Hernández, J., (2020) afirma que los síntomas más frecuentes de la depresión postparto son: tristeza; cambios de apetito; irritabilidad y agitación; sentimientos de inutilidad o culpa; ausencia de placer, insomnio; perdida de energía y fatiga; ansiedad; desconexión; pérdida de concentración; intentos de suicidio; dificultades para realizar tareas domésticas o laborales; incapacidad de cuidar del bebé o de sí misma y sentimientos negativos hacía el bebé.

Guerra Rivas (2020) afirma que el cuadro clínico que suelen presentar las mujeres con depresión postparto, no se diferencia cualitativamente de los otros episodios depresivos que se presentan en contextos distintos al postparto. Este autor afirma que, para poder diagnosticar una depresión postparto, desde el punto de vista psicológico, se deben de presentar los siguientes síntomas: estado de ánimo deprimido durante todo el día, desmotivación por realizar las actividades cotidianas, perdida o aumento del peso corporal, insomnio, dificultades con los procesos psicológicos relacionados con el pensamiento.

Vera Torres (2020) plantea que los factores psicosociales y biológicos, que pueden afectar el estado de ánimo de la mujer, después del parto. Malpartida Ampudia, (2020) señala que conjuntamente con el tratamiento farmacológico y psicoterapéutico, la paciente que presenta depresión postparto, debe seguir las siguientes recomendaciones, para mejorar su calidad de vida: mantener el optimismo; conservar y desarrolla la salud física; y mental, mediante la práctica sistemática de actividades físicas con fines profilácticos y /o terapéuticos; llevar un régimen de vida saludable; ir asumiendo de forma progresiva y gradual las responsabilidades que tenía antes del parto; aumento de la autoestima al aceptarse a uno mismo; expresar las emociones; Seguir el tratamiento impuesto por el psicoterapeuta y el resto del equipo interdisciplinario.

Samaniego Ávila, (2020) señala que durante el embarazo las posibilidades de riesgo de la depresión pueden aumentar por las siguientes causas: Abusos del consumo de drogas y alcohol, familiares que padecen de enfermedades mentales, falta de apoyo emocional de familiares y amistades, incremento del nivel de ansiedad sobre él bebe, dificultades presentadas por la paciente en partos anteriores, dificultades económicas para enfrentar el embarazo y el nacimiento del bebe y el embarazo precoz.

Según Morales, N., (2020) señala que la Psicoprofilaxis Obstétrica, tiene como objetivo instruir a las futuras madres sobre los peligro o complicaciones que pueden tener durante el parto normal, lo que conllevaría a la realización de una cesaría. Este autor señala que los programas de Psicoprofilaxis Obstétrica, contribuyen a: la disminución de la ansiedad, 
mediante el incremento de la autoestima y la actitud positiva ante los problemas presentado por la mujer durante y después del parto, una mejor utilización de las técnicas respiratorias con los músculos del diafragma de masaje y relajación que posibilitan una mejor respuesta a las contracciones uterinas durante el parto, mediante una mayor dilatación vaginal, lo que favorece la disminución de la fase de dilatación con relación al promedio normal, la duración de la fase expulsiva del bebe y el tiempo de duración del parto, lo que favorece la actitud para la lactancia natural y con ello aumenta la calidad de vida del binomio madre- bebe.

\section{Metodología}

\section{Desarrollo}

La presente investigación es de tipo explicativadescriptiva, la que se desarrolló en el periodo comprendido entre agosto del 2019 y enero del 2020, se consto con una muestra de 30 madres adolescentes que presentaron depresión postparto en los recintos de la parroquia San Juan de Juana de Oro, Los Balzares, la Pitaya y Las Marías, donde predomina la actividad agrícola, del cultivo del arroz, banano y el cacao. A continuación, detallamos la conformación de la misma, en los diferentes grupos de edades.

Tabla 1 Muestra seleccionada para el estudio

\begin{tabular}{|c|c|c|}
\hline Edad & $\mathbf{f}$ & $\%$ \\
\hline 15 & 10 & 33.3 \\
\hline 16 & 8 & 26.6 \\
\hline 17 & 7 & 23.3 \\
\hline 18 & 5 & 16.8 \\
\hline Total & 30 & 100 \\
\hline
\end{tabular}

Elaborado por: Autoras

\section{Técnicas y procedimientos}

El análisis bibliográfico, se llevó a cabo mediante la utilización de los método analítico- sintético, que permitieron realizar el análisis de las diferentes fuentes documentales relacionadas con la depresión postparto y en la aplicación de los instrumentos, que permitieron la obtención y recolección de datos cualitativos, a través de la descomposición de las variables dependiente e independiente, que conformaron el tema objeto de estudio, en los indicadores, que a su vez posibilitaron el análisis e interpretación de los resultados y descomponer la propuesta en múltiples relaciones $\mathrm{y}$ componentes, que posibilitaron sintetizar las características generales que se establecieron entre los elementos que la componen, a través del conocimiento objetivo que se investigó.

Por otro lado, la Inducción-deducción, permitió adentrarnos en toda la parte teórica del tema; hacer generalización sobre la problemática y arribar a las conclusiones. Ambos métodos se complementaron en una unidad dialéctica como forma de razonamiento teórico, por medio del cual se pasa del conocimiento particular a un conocimiento más general y viceversa, y para establecer las conclusiones lógicas que determinaron los elementos teóricos - prácticos generales, a partir del carácter social y objetivo del proceso de investigación educativa. Se aplico una encuesta de 11 ítems a las 30 madres adolescentes que fueron objeto de investigación, con el objetivo de identificar los síntomas de la depresión postparto para determinar la efectividad de la estrategia educativa, para aplicar el programa de Profilaxis Obstétrica para la disminución de la depresión postparto. 


\section{Resultados}

Tabla 2 Resultados de la encuesta

\begin{tabular}{|c|c|c|c|c|c|c|}
\hline \multirow[t]{2}{*}{ Items } & \multicolumn{2}{|c|}{ Siempre } & \multicolumn{2}{|c|}{ A veces } & \multicolumn{2}{|c|}{ Nunca } \\
\hline & f & $\%$ & f & $\%$ & f & $\%$ \\
\hline ¿Se siente deprimida durante la mayor parte del dia después del parto? & 30 & 100 & 0 & 0 & 0 & 0 \\
\hline $\begin{array}{l}\text { ¿Se sintió interesada y motivada por realizar las actividades domésticas y } \\
\text { laborales que desarrollabas antes del parto? }\end{array}$ & 0 & 0 & 0 & 0 & 30 & 100 \\
\hline $\begin{array}{l}\text { ¿Has notado algún aumento o pérdida significativa de peso después del } \\
\text { parto? }\end{array}$ & 30 & 100 & 0 & 0 & 0 & 0 \\
\hline ¿Duermes normalmente lo acostumbrado? & 0 & 0 & 0 & 0 & 30 & 100 \\
\hline ¿Sientes que te cansas o agota con frecuencia? & 30 & 100 & 0 & 0 & 0 & 0 \\
\hline ¿Se sientes segura de si misma y de tu bebe? & 0 & 0 & 0 & 0 & 30 & 100 \\
\hline ¿Se sientes desesperada o agitada en la mayoría de las ocasiones? & 30 & 100 & 0 & 0 & 0 & 0 \\
\hline ¿Presentas dificultades económicas en tu hogar? & 30 & 100 & 0 & 0 & 0 & 0 \\
\hline ¿Tienes problema con tu pareja? & 30 & 100 & 0 & 0 & 0 & 0 \\
\hline ¿Su familia le brinda el apoyo necesario? & 0 & 0 & 0 & 0 & 30 & 100 \\
\hline ¿Consume bebidas alcohólicas? & 0 & 0 & 30 & 100 & 0 & 0 \\
\hline
\end{tabular}

Elaborado por: Autoras

\section{Análisis e interpretación de los resultados}

En la categoría de siempre el $100 \%$ de las madres encuestadas respondieron que:

$>$ Se siente deprimidas durante la mayor parte del día después del parto

$>$ Después del parto han experimentado aumentos y disminución del peso

$>$ Se cansan y agotan con frecuencia

$>$ Se sienten desesperadas y agitadas

$>$ Presentan dificultades económicas en el hogar

En la categoría de a veces, el 100\% de las madres adolescentes encuestadas respondieron que

$>$ Consumen bebidas alcohólicas

$>$ En la categoría de nunca el 100\% de las madres encuestadas respondieron que:

$>$ Se sintieron interesada y motivada por realizar las actividades domésticas y laborales que desarrollaban antes del parto

$>$ Duermen normalmente lo acostumbrado

$>$ Se sienten segura consigo misma y con su bebe

$>$ Su familia le brinda el apoyo necesario.
Los resultados obtenidos de la aplicación de la entrevista nos permiten afirmar que el $100 \%$ de las madres adolescentes que fueron encuestadas presentan síntomas de depresión postparto.

Propuesta de una Estrategia educativa, para la aplicación del programa de psicoprofilaxis obstétrica a madres adolescentes que presentan depresión postparto en la parroquia San juan cantón San Francisco de Puebloviejo.

\section{Fundamentación de la Estrategia Educativa.}

Dentro de los aspectos potencializadores desde el punto de vista educativo dentro del contexto del programa de psicoprofilaxis obstétrica, se debemos tener en cuenta la concepción holística de este proceso, la estrategia educativa para la aplicación del programa profiláctico obstétrico, se sustenta en un enfoque integrador $d$ los aspectos cognitivo y socio afectivo, con lo instructivo educativo y desarrollador, como requisitos psicológicos y pedagógicos esenciales para el adecuado funcionamiento del mismo, a partir de las características individuales de las madres adolescentes que 
presentan depresión postparto, según los resultados de las encuestas que fueron aplicadas y la organización y selección de los contenidos de la actividades del programa, mediante el establecimiento de indicadores que estén relacionados con el tratamiento psicoprofiláctico obstétrico, en las etapas de planificación, organización y dirección del programa psicoprofiláctico.

Etapas del plan de acciones estratégicas para la aplicación del programa psicoprofiláctico obstétrico a las madres adolescentes que presentan depresión postparto.

\section{Diagnóstico:}

Selección de instrumentos, a partir de los síntomas de depresión postparto presentados por las madres adolescentes, teniendo en cuenta las potencialidades y dificultades para lo que se debe establecer un orden jerárquico de los problemas detectados en correspondencia con los objetivos propuestos.

\section{Etapa de Planificación y organización:}

Determinación del contenido de las actividades a desarrollar en el programa psicoprofiláctico obstétrico, a partir de las características anatomo fisiológicas y psicológicas de las madres adolescentes, mediante la ejecución de actividades que pueden aplicarse en las edades de los sujetos que conforman la muestra.

Etapa de ejecución del plan de acciones.

Planteamiento de actividades educativas, ejercicios físicos, técnicas de actividades respiratorias y de relajación del sistema neuromuscular, que contribuyan a la profundización y actualización de los conocimientos de las madres adolescentes que presentan depresión postparto que permitan practicas saludables para elevar la calidad de vida de la madre y él bebe, a partir de las dimensiones: actitudinal emocional (ayuda al recién nacido, mediante el vínculo afectivo y la alimentación del bebe) y comportamental (desarrollo de habilidades para enfrentar el proceso de postparto, y comunicarse $y$ relacionares con los demás)

\section{Formas de instrumentación de la Estrategia Educativa en el contexto comunitario.}

El tipo de actividad a desarrollar son los talleres de capacitación a las madres adolescentes en las instalaciones de las comunidades de la parroquia San Juan objeto de estudio.

Las etapas de aplicación se describen a continuación.

> Familiarización: tiempo de duración 3 meses, dos veces a la semana en un tiempo de 60 minutos.

$>$ Desarrollo: tiempo de duración 3 meses, tres veces a la semana en un tiempo de 90 minutos.

$>$ Control final.

\section{Valoración de la efectividad de la Estrategia}

Para la valoración de estos indicadores en la etapa de control o final se recurrió a la aplicación de los mismos instrumentos de la etapa de constatación, instrumentos que en su interpretación cualitativa permiten comprender la efectividad de la Estrategia Educativa en la muestra seleccionada dentro del programa psicoprofiláctico obstétrico a las madres adolescentes que fueron objeto de investigación.

En la encuesta aplicada a las madres adolescentes, se evidencio en un incremento de la autoestima, la seguridad en sí misma y en su bebe y la disposición por comenzar a realizar las actividades que habitualmente desarrollaban antes del parto, superando así las expectativas para contribuir a elevar la calidad de vida de las mismas. 
A partir de los resultados obtenidos con la aplicación de la estrategia educativa, se consideró pertinente la valoración de los indicadores eficacia, funcionalidad e impacto, en tal sentido:

La eficacia caracterizada por la obtención de resultados cada vez más acertados con relación a los objetivos previstos en la solución del problema; manifestado a partir de la relación de los análisis de los indicadores y acciones estratégicas elaboradas para el proceso educativo dentro del programa psicoprofiláctico obstétrico y específicamente para elevar la autoestima y la calidad de vida.

La funcionalidad se destaca el grado en que se valoró la pertinencia del proceso de validación de los indicadores independientemente de los resultados obtenidos, y en qué medida resultó oportuna la aplicación de estos en las actividades dentro del programa psicoprofiláctico obstétrico en las madres adolescentes que fueron objeto de estudio, la funcionalidad se pudo constatar, a través del análisis de las relaciones establecidas entre los sujetos que intervinieron en la investigación.

El impacto se verificó en la medida en que la validación permitió valorar el efecto que tienen los resultados de la aplicación de la Estrategia Educativa, es decir, el impacto está dado en el grado en que las madres adolescentes se integran de forma activa a la sociedad dentro del proceso educativo en el programa psicoprofiláctico obstétrico, como principal intencionalidad en este proceso. El impacto de este proceso de validación se ha expresado esencialmente en el ámbito psicológico en tanto se ha incrementado la autoestima y la calidad de vida, lo que se evidencio en la incorporación activas de las madres adolescentes que fueron objeto de investigación, a las actividades que se desarrollan en las comunidades.

\section{Conclusiones}

Luego del análisis e interpretación de los resultados y de la verificación de las hipótesis estamos en condiciones de dar las conclusiones de la investigación.

El tratamiento de Psicoprofilaxis obstétrica influyo positivamente en la disminución de la depresión postparto en las madres adolescentes del sector La Aventura Cantón Babahoyo Provincia de Los Ríos durante el primer semestre del año 2015, lo que se evidencia con los resultados obtenidos en las observaciones a las capacitaciones.

Los resultados de las encuestas evidenciaron que los síntomas de depresión postparto que presentan las madres adolescentes del sector la Ventura del cantón Babahoyo, están dados por problema socio afectivos.

Los resultados obtenidos en la investigación demuestran que las madres adolescentes que padecen de depresión postparto en el sector la Ventura del cantón Babahoyo no habían sido sometidas a un tratamiento psicoprofiláctico obstétrico durante esta etapa.

Los resultados obtenidos en la investigación evidencian que el diseño de la capacitación sobre Psicoprofilaxis obstétrica influyo favorablemente en la disminución de la depresión posparto de las madres adolescentes del sector la Ventura del cantón Babahoyo.

Los resultados obtenidos mediante la aplicación de la guía de observación evidencian que el tratamiento Psicoterapéutico obstétrico fue efectivo para en disminución de la depresión postparto en las madres adolescentes del sector la Ventura del cantón Babahoyo. 


\section{Recomendaciones}

Luego de las conclusiones de esta investigación proponemos las siguientes recomendaciones.

Dar a conocer los resultados de esta investigación a las autoridades de la salud de la provincia Los Ríos y el Cantón Babahoyo, para que se generalicen en estos resultados,

Que se le dé continuidad a esta investigación a través de los Programas de Vínculo con la Sociedad, como una alternativa de generalizar los resultados en otras comunidades de la localidad de Babahoyo,

Presentar los resultados de esta investigación en eventos científicos relacionados con la carrera de Obstetricia o de la salud.

Divulgar los resultados obtenidos en esta investigación en los medios locales como: la radio y la televisión.

\section{Referencias Bibliográficas}

David, D. \&. (2020). Caracterización de la depresión postparto en madres de 20 a 40 años Riobamba 2019 (Bachelor's thesis, Universidad Nacional de Chimborazo).

Guerra Rivas, G. A. (2020). Depresión postparto y su influencia en el núcleo familiar (Bachelor's thesis, Babahoyo: UTB).

Hernández, J. J. (2020). Factores asociados a depresión posnatal en puérperas de Colombia, según escala de Edimburgo. Ciencia e Innovación en Salud.

Horowitz, J. A. (2020). Relación entre detección de depresión postparto, síntomas de severidad y períodos de tratamiento. Revista Horizonte de Enfermería, 27(1), 4858.

Malpartida Ampudia, M. K. (2020). Depresión postparto en atención primaria. Revista Médica Sinergia, 5(02). 355-355.

Morales, N. V. (2020). Beneficios y dificultades de la psicoprofilaxis obstétrica en gestantes de la comunidad de Progreso, Guayas. Pro Sciences: Revista de Producción, Ciencias e Investigación, 4(33), 30-40.

Samaniego Ávila, V. C. (2020). Factores asociados a la depresión postparto en gestantes ingresadas en el área de ginecología de un hospital de la ciudad de Guayaquil, año 2019.

Vera Torres, P. E. (2020). Depresión postparto en madres que asisten al centro de salud no. 1 Pumapungo-Cuenca y su relación con las variables sociodemográficas (Bachelor's

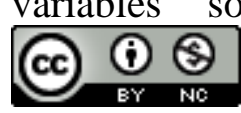

de Creative Commons Reconocimiento-No Comercial 4.0 Internacional. Copyright (c) Georgina Azucena Romero Torres, Josselin Cherrez Romero y Genesis Thayly Barros Monserrate 
